\title{
Rheumatoid arthritis in twins: a study of aetiopathogenesis based on the Australian Twin Registry
}

N Bellamy, D Duffy, N Martin, J Mathews

\begin{abstract}
The 1980 cohort of the Australian Twin Registry contains 3808 pairs of twins, 258 of whom self reported a diagnosis of rheumatoid arthritis (RA) in one or both subjects. Seventy two pairs were lost to follow up by 1990 . The remaining 186 pairs received a self administered questionnaire, followed, if necessary, by telephone interviews to them, their general practitioners, and their specialists. Twenty discordant and three concordant pairs of twins were verified as having RA. The prevalence of RA in this sample was $0.40 \%$. There was an $89 \%$ false positive rate for the self reported diagnosis of RA. Pairwise concordance percentages for RA were as follows: monozygotic $\mathbf{2 1 \%}$ (95\% confidence interval $(\mathrm{CI})=6$ to 44$)$, dizygotic $0 \%(95 \%$ $\mathbf{C I}=\mathbf{0}$ to 25$)$. It was concluded that: $(a)$ there is a high false positive rate in self reporting RA; $(b)$ the prevalence of RA in Australia may be less than the $0.8-1.0 \%$ often quoted; and (c) genetic factors play some part in the aetiopathogenesis of RA but do not account entirely for its determination.
\end{abstract}

The part played by genetic factors in the aetiopathogenesis of rheumatoid arthritis (RA) has been the subject of previous study. In particular, there have been a number of twin studies, notably those evaluating one or more pairs of twins, ${ }^{1-8}$ and the two population based studies by the Arthritis and Rheumatism Council ${ }^{9}$ and Aho et al. ${ }^{10}$ Although a combination of epidemiological surveys, family and twin studies, and observations on the relation between disease and HLA genotype has resulted in a better understanding of RA, nevertheless, the relative contribution of genetic and environmental factors remains obscure. Previous studies have been limited by one or more factors including small sample size, highly selected patients, and a lack of adequate diagnostic information. To further investigate the aetiopathogenesis of RA, we have conducted a study of twins with RA in Australia. In contrast with previous studies, we have used a large population based sample and applied strict criteria in defining, the presence of RA.

\section{Patients and methods}

\section{SUBJECTS}

The study used the Australian National Health and Medical Research Council Twin Registry. This is a volunteer registry started in 1978 . Twins were recruited through schools, com- munity groups, and by media advertising throughout Australia. About 16000 pairs of twins of all types and ages, or about $10-15 \%$ of the expected number, are registered. From November 1980 to March 1982 a questionnaire was sent to all 5967 adult ( $>18$ years) pairs then registered. Of this group, 3808 twin pairs returned completed questionnaires (a pairwise response rate of $64 \%$ ). The questionnaire was extensive and included items on age, sex, zygosity, birth order, tobacco use, psychological traits, and a number of physical and psychiatric symptoms. The disease checklist was completed for 'self', 'cotwin', and 'other family members', RA being one of the 13 diseases or conditions listed. Zygosity of the twins was diagnosed by response to a number of items, supplemented in ambiguous cases by examination of photographs sent in by the twins. This method has been shown to be at least $95 \%$ accurate in several other studies. ${ }^{11}$ On the original questionnaire, a diagnosis of ' $R A$ ' in twin or cotwin, or both, was self reported in 258/3808 pairs. In addition to the specific question regarding RA, there were two additional questions asking about the occurrence of 'stiff joints in the morning' and 'arthritis or rheumatics'. Only twin pairs responding positively to the RA question were included in this study. By 1990, either one or both subjects in 72/258 ' $\mathrm{RA}$ ' pairs were lost to follow up for one of the following reasons: deceased; refused to continue as a active registrant; or untraceable. The remaining 186 pairs formed the focus of this research, although an attempt was made to phone as many of the survivors from the 72 'unavailable' pairs to verify their musculoskeletal status.

\section{CLINICAL PROFILE QUESTIONNAIRE}

The following method was used to investigate the musculoskeletal status of the 186 eligible pairs. A 19 component self administered clinical profile questionnaire was developed and formatted. Most components contained multiple items. The components probed the patient's demographic profile, musculoskeletal symptoms and signs, history of gastrointestinal investigation, beneficial and adverse reactions to previous and current treatment with antirheumatic drugs, major medical or surgical problems, concurrent drug treatment for nonmusculoskeletal problems, obstetric history (where applicable), relationship between disease activity and gestation (where applicable), month, year and joint of initial onset of RA, Steinbrocker functional class, ${ }^{12}$ a record of every joint ever affected by arthritis, and the 


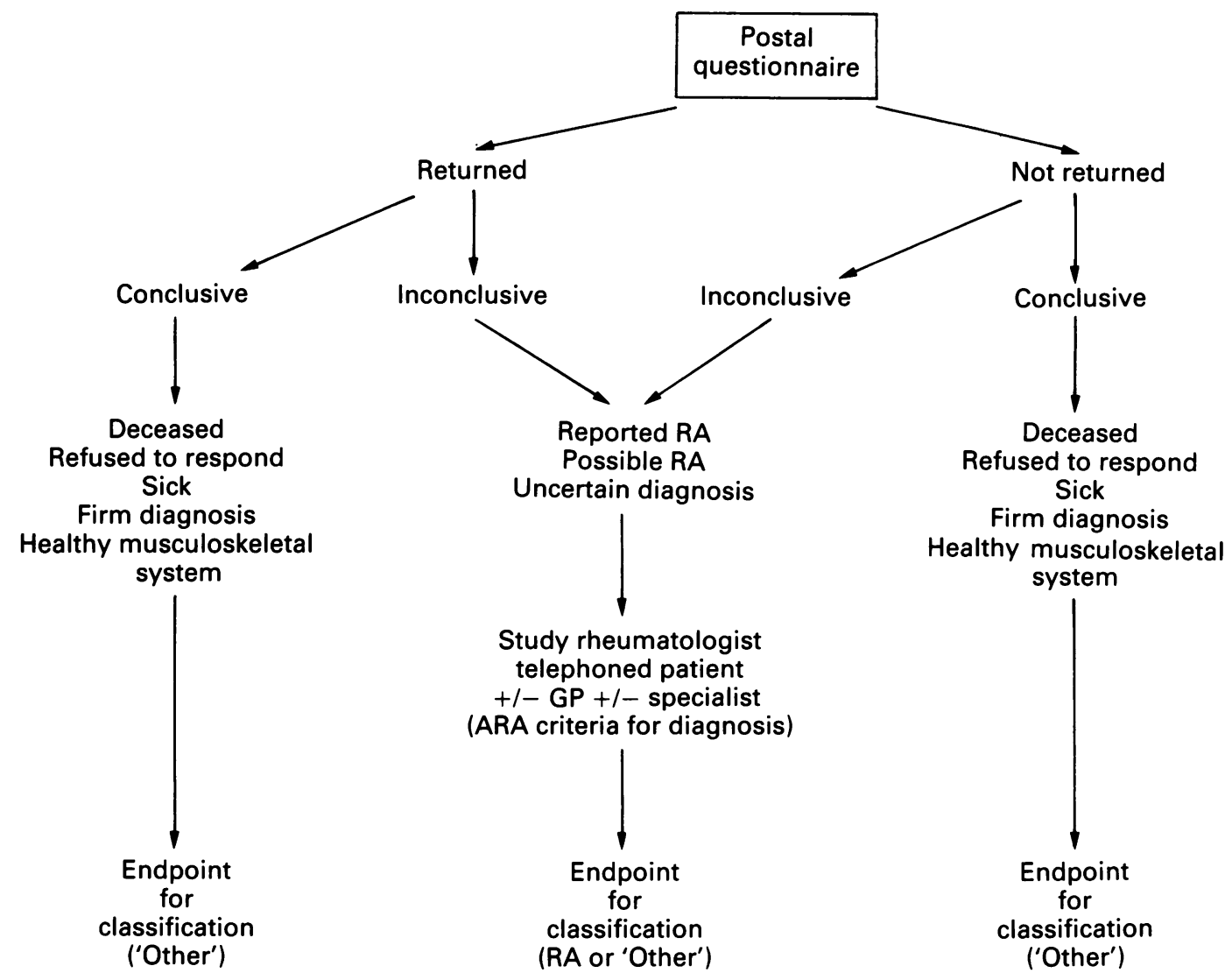

Survey strategy for diagnostic verification. Final classification was by subsequent telephone tracing/interview. $R A=$ rheumatoid arthritis; $G P=$ general practitioner; $A R A=$ American Rheumatism Association.

revised 1987 American Rheumatism Association (ARA) classification criteria for RA. ${ }^{13}$ The ARA criteria were completed by the patient's general practitioner or specialist, or both, following the patient's written consent. Skip options were provided, where necessary, to lead respondents through irrelevant portions of the clinical profile questionnaire and a truncation procedure was used to terminate the response process for subjects unaffected by RA.

\section{SURVEY STRATEGY}

The figure shows the basic strategy, which used a postal contact, followed where necessary by telephone interview. The clinical profile questionnaire was sent to 186 pairs of twins in June 1990. Commencing three weeks later, returned clinical profile questionnaires were classified as either 'conclusive' or 'inconclusive'. Conclusive clinical profile questionnaires were those which categorised the respondent in one of the following groups: deceased; refused to respond; sick and unable to respond; firm musculoskeletal diagnosis other than $\mathrm{RA}$; or healthy musculoskeletal system (no joint problems). Inconclusive clinical profile questionnaires were those returned by subjects reporting either definite RA, possible RA, or in whom the diagnosis of arthritis was uncertain. Patients in all three 'inconclusive' categories required further investigation by the study rheumatologist (NB). In those instances where the clinical profile questionnaire was not returned, a panel of experienced interviewers, working in association with and including the study rheumatologist, phoned non-respondents to obtain key diagnostic information and encourage the return of the original clinical profile questionnaire. Where necessary, subjects were contacted through their cotwin or sent replacement questionnaires.

In some instances clinical profile questionnaires were completed over the phone. Phone calls were placed at different times of day, and different days of the week if initial attempts were unsuccessful. Two axioms guided the interviewers: (a) subjects who had never experienced joint pain, swelling, or stiffness, past or present, were regarded as healthy from a musculoskeletal standpoint; and $(b)$ a diagnosis of RA was considered untenable in patients who had never experienced any joint swelling even though they might have experienced pain or stiffness, or both, in their joints. Following telephone interviews and receipt of any outstanding clinical profile questionnaires data from respondents were again classified as being conclusive or inconclusive using the aforementioned criteria (fig). Pursuit of the diagnosis in those subjects whose data were classified as inconclusive was carried out exclusively by the study rheumatologist. The process involved reviewing the clinical profile questionnaire, clarifying any ambiguity, and correcting any errors or deficiencies. Thereafter, the study rheumatologist spoke with the twin and cotwin regarding the nature of their musculoskeletal symptoms and signs and the basis for any diagnosis. In essence, an abbreviated history 
was taken over the phone. In addition to considering the 1987 ARA criteria for the classification of RA, enquiries were made about any alternative musculoskeletal diagnosis, particularly that of psoriatic arthritis.

Following receipt of the patient's informed consent, the study rheumatologist contacted the patient's general practitioner and in those instances when the patient had seen a specialist, also the patient's rheumatologist or orthopaedic surgeon. A checklist of ARA criteria was provided to the patient's doctor, either via the patient or sent directly, or discussed over the phone. In some instances the clinical profile questionnaire was returned with the ARA checklist already completed by the patient's doctor. In reaching a diagnosis any available serological or radiographic data were noted and the diagnostic opinion of the attending doctor considered. For those subjects whose diagnosis was uncertain, an attempt was made to establish the true musculoskeletal diagnosis, or if this proved impossible then the subject was categorised as 'arthritis NYD' 'unclear diagnosis' (fig). It should be noted in these patients that although the exact diagnosis was unclear, it was almost certainly not RA. Owing to the wide geographical dispersal of twins throughout Australia, it was possible for the study rheumatologist to physically examine only two of the twins (a concordant pair of identical twins). Data were analysed using the statistical package for the social sciences. ${ }^{14}$

\section{Results}

Table 1 gives the final status in 1990 of the 516 subjects from the 1980 cohort. There were 168 subjects lost to follow up by death or withdrawal from the Australian Twin Registry, 136 between 1980 and last contact at 1989, and a further 32 up to the time of the survey. There were 12 subjects initially thought lost in 1989 who were recontacted and interviewed by telephone (none of whom were diagnosed as having RA). A further 12 subjects contacted refused to cooperate, whereas four were too ill to be interviewed-that is, equivalent to $96 \%$ cooperation. Overall, however, there was a 10 year follow up rate of $64 \%$ (184 (36\%) subjects from the 1980 cohort failed to complete the 1990 clinical profile questionnaire; table 1). Among the 332 respondents there were 63 (19\%) subjects who were completely healthy from a musculoskeletal standpoint, of whom 53 were unaffected cotwins. On contacting the remaining 10 subjects who had self reported a diagnosis of RA on the 1980 questionnaire, it was found that they had marked the original questionnaire erroneously and had never had arthritis.

Twenty six patients with RA fulfilling the 1987 ARA criteria were detected in the 1990 sample, four having apparently developed the disease since 1980. Setting the 1990 diagnosis as a 'gold standard' and the 1980 diagnosis as the test standard, it can be calculated that the 1980 responses had a sensitivity for RA of $85 \%$, a specificity of $53 \%$, a false positive rate of $89 \%$, and a false negative rate of $2 \%$ (table 2 ). A false positive is a patient who self reported RA in 1980 but in whom the ARA criteria could not be fulfilled by 1990 . A false negative is a patient who denied RA in 1980 but by 1990 had fulfilled the ARA criteria for RA. Of the four false negative patients, two certainly and two probably had an onset of RA after 1980 . If the diagnostic information provided by the cotwin was also considered, then when the twin reported RA and the cotwin 'confirmed' the diagnosis, this was more likely to denote a genuine case (Cohen's $x=0.26,95 \%$ confidence interval $(C I)=0.11$ to 0.41$)$. Using this approach the sensitivity of the self reported diagnosis was $50 \%$, the specificity $89 \%$, and the positive predictive value $25 \%$. It should be noted that subjects lost to follow up did not differ in the prevalence of 'cotwin confirmed' disease from those included in the analysis.

Of the 26 subjects with RA there were 23 women and three men (relative risk female $v$ male 4.3 ). They had a mean age of 43.5 years (range 25-71), a mean age of onset of 39 years (range 16-70), and disease duration of 15 years
Table 1 Classification in 1990 of all 516 individual twins reporting 'rheumatoid arthritis' (RA) in 1980 health and lifestyle questionnaire

\begin{tabular}{lcc}
\hline Diagnosis & $\begin{array}{l}\text { No of positive } \\
\text { respondents }\end{array}$ & $\begin{array}{c}\text { Percentage of } \\
\text { returns }\end{array}$ \\
\hline Rheumatoid arthritis & 26 & $7 \cdot 8$ \\
Connective tissue disease & 2 & $0 \cdot 6$ \\
Psoriasis +rheumatism & 4 & $1 \cdot 2$ \\
Palindromic rheumatism & 4 & $1 \cdot 2$ \\
Osteoarthritis & 95 & $28 \cdot 6$ \\
Gout & 5 & $1 \cdot 5$ \\
Arthralgia & 59 & $17 \cdot 8$ \\
Miscellaneous rheumatism & 47 & $19 \cdot 2$ \\
Normal & 63 & $8 \cdot 1$ \\
Unclear diagnosis* & 27 & \\
Clinical profile & & $100 \cdot 0$ \\
$\quad$ questionnaire returned & & \\
(total) & $332(64 \%) \dagger$ \\
Clinical profile & & \\
questionnaire not & & \\
done (total) & $184(36 \cdot 0 \%) \dagger$ \\
Total no of subjects & $516(100 \%) \dagger$ \\
\hline in 1980 cohort
\end{tabular}

${ }^{*}$ Although the exact diagnosis was unclear in these 27 subjects, it was almost certainly not RA

†Percentages of subjects from 1980 cohort.
Table 2 Agreement of 1980 self reported diagnosis of rheumatoid arthritis (RA) with 1990 follow up diagnosis

\begin{tabular}{lll}
\hline & $\begin{array}{l}\text { Actual } R A \\
\text { in } 1990\end{array}$ & $\begin{array}{l}\text { No actual } R A \\
\text { in } 1990\end{array}$ \\
\hline $\begin{array}{l}\text { Reported 'rheumatoid' } \\
\text { in 1980 }\end{array}$ & 22 & $167 \dagger$ \\
$\begin{array}{l}\text { Denied 'rheumatoid' } \\
\text { in 1980 }\end{array}$ & $4^{*}$ & 189 \\
Total & 26 & 356
\end{tabular}

*These are four subjects who either developed the disease since 1980 , or did not report the date of onset.

†These patients never had RA.

These figures exclude 16 subjects: 12 refusals and four deaths.

Table 3 Monozygotic and dizygotic twin concordances for 'true' rheumatoid arthritis

\begin{tabular}{llll}
\hline $\begin{array}{l}\text { Type of } \\
\text { twin }\end{array}$ & $\begin{array}{l}\text { Concordant } \\
(++)\end{array}$ & $\begin{array}{l}\text { Discordant } \\
(+-)\end{array}$ & $\begin{array}{l}\text { Concordant } \\
(-)\end{array}$ \\
\hline Monozygotic* $^{*}$ & 3 & 11 & 1785 \\
Dizygotic $^{*}$ & 0 & 9 & 2000
\end{tabular}

*Numbers refer to pairs. 
Table 4 Estimated prevalence in those over 15 years of rheumatoid arthritis using the 1983 Australian Bureau of Statistics $(A B S)$ derived population structure and age and sex standardisation of our prevalence datał

\begin{tabular}{|c|c|c|c|c|c|}
\hline & \multicolumn{5}{|c|}{ Age band (in 1983) years } \\
\hline & $15-24$ & $25-44$ & $45-64$ & $65+$ & Over 15 \\
\hline $\begin{array}{l}\text { Birth cohort } \\
\text { Our number } \\
1980 \text { cohort (women) } \\
1980 \text { cohort (men) } \\
\text { Prevalence (men and women)* } \\
\text { Population cohort prevalence } \\
\text { ABS cohort }\left(\times 10^{3}\right)\end{array}$ & $\begin{array}{l}1968-59 \\
0 / 18 \\
0 / 840 \\
0 / 582 \\
0 \%(0-0 \cdot 06 \%) \\
2588 \cdot 0\end{array}$ & $\begin{array}{l}1939-58 \\
12 / 152 \\
11 / 2673 \\
1 / 1537 \\
0 \cdot 3 \% \\
0 \cdot 3 \% \\
4514 \cdot 1\end{array}$ & $\begin{array}{l}1919-38 \\
10 / 158 \\
8 / 1057 \\
2 / 463 \\
0 \cdot 7 \% \\
0 \cdot 7 \% \\
2914 \cdot 0\end{array}$ & $\begin{array}{l}<1919 \\
1 / 42 \\
1 / 299 \\
0 / 161 \\
0 \cdot 2 \% \\
0 \cdot 8 \% \dagger \\
1407 \cdot 1\end{array}$ & $\begin{array}{l}<1969 \\
22 / 370 \\
4872 \\
2744 \\
0 \cdot 4 \% \dagger \\
11423 \cdot 2\end{array}$ \\
\hline
\end{tabular}

*Owing to the excess of women volunteers, this is inflated over the true population prevalence in each age cohort.

+Using the prevalences from the 45-64 year old cohort as an estimate for the true prevalence in the $65+$ year old cohort.

$¥$ Australian Health Survey. 1983: Appendix 3 Australian Bureau of Statistics. Canberra 1983 (catalogue No 4311.0).

(range 2-31). Twenty were seropositive and five were seronegative for IgM rheumatoid factor (one unknown). The number of ARA criteria fulfilled were as follows: four criteria 6 , five criteria 7 , six criteria 6 , and seven criteria 6 (one other patient had at least four criteria-symmetric polyarthritis, no psoriasis, prolonged morning stiffness, no nodules, rheumatoid factor status unknown, recovering from recent bilateral mastectomy for carcinoma). Steinbrocker class was as follows: class $1=4$, class $2=20$, class $3=2$, and class $4=0$.

The 26 patients with RA were distributed in 23 pairs: 20 discordant and three concordant. There were 14 monozygotic pairs and nine dizygotic pairs (table 3 ). The prevalence of RA in the twin sample (in 1980) was $0.3 \%$; there was a marginal difference between monozygotic and dizygotic pairs (Fisher's exact test, $\mathrm{p}=0.05$ ). The pairwise concordance percentage was $21 \%$ among monozygotic twins and $0 \%$ among dizygotic twins. The expected numbers of concordant pairs were calculated separately for monozygotic and dizygotic twin pairs. The monozygotic twins were significantly more concordant for RA than dizygotic twins: Cohen's $x_{M Z}=0.37(0.04$ to 0.69$) ; x_{\mathrm{DZ}}=-0.01$ to $0 \cdot 00$ ).

The 1983 Australian Bureau of Statistics health survey derived structure was used to standardise our prevalence data for age and sex. Four age bands $(15-24,25-44,45-64,65+$ years) were used in the standardisation (table 4). The estimated adult prevalence (age $>15$ years) of RA in 1980 was $0.35 \%$. No data was available for the 15-24 year age band. The crude and directly standardised prevalences of RA were similar $(25-44$ years $=0 \cdot 3 \%, 45-64$ years $=0 \cdot 7 \%)$. The crude prevalence of RA was lower in the over 65 year old age group at $0.25 \%$, but with an upper (likelihood based) $95 \%$ of $0.95 \%$. Assuming this decline was not due to differential mortality, but rather to the smaller cohort size, we have used the 45-64 year old rates to estimate a standardised prevalence of $0.8 \%$ for the over $65 \mathrm{~s}$, and thus an overall adult standardised prevalence of $0.4 \%$.

\section{Discussion}

Since 1938 a variety of sampling techniques have been used in twin studies of RA. ${ }^{1-10}$ Although case reports (single or multiple) provide descriptive information, they are of limited epidemiological value as they have no population base. ${ }^{1-8}$ The method of ascertaining twins within a population of subjects affected by RA and then examining cotwins to establish their musculoskeletal status provides a different estimate of risk. ${ }^{9}$ Aho et al have used an unlimited representative sampling method linking two national registers: the Finnish Twin Cohort and the Sickness Insurance Register. ${ }^{10}$ There are a number of factors to be considered when reviewing twin studies of RA. Studies before 1959 were conducted before the development of the ARA diagnostic criteria ${ }^{15}$ and the accuracy of the musculoskeletal diagnosis is indeterminate. Two major studies have been conducted between 1959 and 1987, although the application of the ARA criteria in diagnosis is uncertain. There have been no previous studies using the revised 1987 classification criteria which we have used here. The capricious nature of $R A$ requires that account be taken, not only of subjects with persistent chronic $R A$, but also of those who after a short illness go into remission, and those subjects whose disease does not begin until later in life. Ideally, a decision about RA status would not be made until the point of death, but clearly this is impractical. Studies which have followed twins over time are preferable to those assessing twins in early life. In our study the follow up time was 10 years. One of the consequences of prolonged monitoring is loss to follow up, which in this study, taking into account all sources (untraceable, too ill to complete the clinical profile questionnaire, death, unable to contact) was $29 \%$. Clinical assessment can be approached in a variety of ways using chart review, individual history, and physical examination, interview of attending medical staff, and review of radiographs and serological results. The long follow up, the high mobility of modern populations, and the wide geographical dispersal of study subjects all hamper the collection of data which are accurate and complete. The twins are widely distributed throughout Australia; some are in urban centres but others are in rural areas, and it was possible for the study rheumatologist to personally interview and examine only two subjects of one of the concordant pairs. Nevertheless, in this study we have used a variety of techniques to obtain essential diagnostic information.

Any effects of lost follow up (non-response bias) are speculative. Bias can be considered in two dimensions: magnitude and direction. A number of factors suggest that any non-response bias was probably small in magnitude: $(a)$ the prevalence of RA in our study $(0 \cdot 40 \%)$ was very 
similar to that in the Australian Bureau of Statistics population based survey $(0 \cdot 32 \%) ;(b)$ loss to follow up was greatest in the oldest cohorts - that is beyond the time at which most patients destined to develop RA would have declared themselves; $(c)$ the loss to follow-up of patients between 1980 and 1990 from the RA sample was exactly replicated in the total sample; and (d) the mean age of onset of RA (39 years) in this group of patients was close to the usually cited mean age of onset (40 years). ${ }^{16} 17$ With respect to the direction of operation, early mortality in patients with severe RA, or high geographical mobility in patients with mild or remittent RA could, respectively, increase or decrease the observed rates for concordance and prevalence. Given that any non-response bias was probably small in magnitude, however, its direction of operation is less important.

Our study differs from that of the Finnish Twin Study in that record linkage was not used or indeed available. Instead, this study was based on a large voluntary nationwide registry. Furthermore, in the Finnish study a diagnosis of RA which qualified a patient for free drugs was based on the two criteria of notification of diagnosis by an attending doctor and approval by an expert adviser. Although the error associated with this method of determination has not been clearly defined, it could be considerable. Whether or not that diagnosis was based on the 1959 ARA criteria ${ }^{15}$ is uncertain from the published report, but the study certainly predated the 1987 ARA criteria which we have used in this study. Furthermore, in the Australian survey the ARA criteria were exactly applied following personal contact of the study rheumatologist with patients with RA, the attending general practitioner, and any rheumatologist or orthopaedic surgeon who had been consulted. In two instances the respondents were personally interviewed and examined by the study rheumatologist. Even for patients without RA, in ambiguous instances the patient was contacted and, where necessary, the opinion of medical staff sought. As a result, we believe that the verification of diagnosis has been more rigorous in this than previous studies.

Our data provide a number of interesting insights into RA. In the first instance it is clear that the self-reporting of RA is unsatisfactory and associated with an extremely high false positive rate. The accuracy of diagnosis is greater when the cotwin confirms the diagnosis reported by the affected twin than when the diagnosis is unconfirmed or negative (Cohen's $x=0.2 \%, 95 \% \mathrm{CI}=0.11$ to 0.41 ). As noted previously, the ATR questionnaire contained items for self reporting on the health status of 'self', 'cotwin', and 'other family members'. Such a contingency offers obvious advantages given the false positive and to a lesser extent the false negative rate that attend self reporting of diagnosis. In particular, this contingency results in increased specificity of diagnosis. It is essential, however, in population based studies that the diagnosis is confirmed using evidence from experts, serology, and radiographs which can be rated against an international standard.
It should be noted that not only do subjects report having $\mathrm{RA}$ when they have a different musculoskeletal problem, but they may also self report RA when they are completely normal.

Our data are compatible with that of other twin studies, indicating that the overall genetic component in RA is small. The risk ratios are higher for monozygotic than dizygotic pairs, further indicating that hereditary factors play at least some part in the aetiopathogenesis of the disease. We had hoped to address issues related to the time of onset, joints of predilection, effects of pregnancy on arthritis, and beneficial and adverse reactions to antirheumatic drugs in concordant twins, but the small number of pairs precluded any meaningful analysis for epidemiological purposes. We did, however, examine the relation between RA and a number of risk factors: age, gender, alcohol intake, smoking, and glandular fever. No significant association was observed, but it should be noted that the sample size is small and a type II error cannot be excluded. The relative risk of RA in women of $4 \cdot 3$ is, however, compatible with the known female:male (3:1) ratio for RA. The part played by environmental factors in the aetiopathogenesis of RA remains undetermined.

In this study the point prevalence of RA was adjusted using the 1983 Australian Bureall of Statistics derived population structure and age and sex standardisation techniques. Although the value of $0.40 \%$ was less than the $0.8-1 \cdot 0 \%$ often quoted, ${ }^{18}$ a number of factors should be considered.

Firstly, given the natural tendency of RA to begin at certain ages more than others, the lifetime prevalence of RA may be higher than the point prevalence estimate as the latter is highly dependent on the age mix of the study patients. Although our sample is not typical of the age structure of the Australian population, the differences are sufficiently small that we do not believe it makes much difference to our prevalence estimates.

Secondly, although RA is a ubiquitous disease, prevalence estimates show some geographical variation and the disease may be less frequent in Australia than in northern Europe.

Thirdly, inclusion in the Australian Twin Registry is voluntary. Although it is thought that about $10 \%$ of Australian twins are currently registered, no data are available on twins not registered to allow comparison. Thus, we cannot comment on the effect of initial enrollment (volunteer bias) on the composition of the sample. As we consider it unlikely that even severe arthritis would preclude patients either from enrolling initially or completing the clinical profile questionnaire, the aforementioned factors are unlikely to have resulted in any important differential loss of subjects.

Lastly, in the 1978 Australian health survey, data were collected on the specific diagnosis of $R A$. The population prevalence of $R A$ in that survey was $0 \cdot 32 \%$. This figure is very similar to the $0.40 \%$ estimate in this study.

Although genetic factors may not be the only or, indeed, the major aetiological determinant of RA, they may be important in disease expression. To date the subjects studied have 
not been HLA typed, and, as a result, we are not able to comment on the relation between HLA haplotype and disease severity. Likewise, there are insufficient monozygotic and dizygotic concordant pairs to make any statement on the comparability of disease (severity, symptomatology) in identical compared with non-identical twins. If genetic factors do not fully explain disease determination, it suggests that environmental factors, either infectious agents, environmental toxins, natural hazards, dietary factors, or some other facet of modern living, may be instrumental. Indeed, if data on the antiquity of RA are to be believed, ${ }^{19}$ it may be necessary to concentrate on environmental factors, which have changed in the last 200 years, on infectious agents, and on the consequence of increased longevity (increased duration of exposure to the environment). Our current data do not allow an adequate assessment of environmental conditions of potential aetiological importance. However, like preceding studies in other populations using different methodologies, we have reached a similar conclusion-that is, genetic factors play at least some part in disease determination.

The authors thank Sue Mason, Ann Eldridge, and Elizabeth Cronin for assistance with telephone interviews. The Australian Twin Registry is supported by the National Health and Medical Research Council.

1 Claussen F, Steiner F. Die Bedeutung der Konstitution für die Erkrankung an Gelenkrheumatismus. Verh Dtsch Ges Inn Med 1938; 50: 299-303.

2 Edstrom G. Klinische Studien uber den chronischen Glen- krheumatismus; 1. Das Erbbid. Acta Med Scand 1941; 108: 398-413.

3 Brandt G, Weihe T A. Polyarthritis rheumatica bei zwillingen. Z Menschl Vereb-u Konstit Lehre 1939; 23: 169-88.

4 Thymann G. Polyarthritis in twins. Acta Genetectica Basel 1957; 7: 148-50.

5 Moesmann G. Factors precipitating and predisposing to rheumatoid arthritis as illustrated by studies in monozygotic twins. Acta Rheumatol Scand 1959; 5: 291-303.

6 Harvald B, Hauge $M$. Hereditary factors elucidated by twin studies. In: Genetics and the epidemiology of chronic diseases. studies. In: Genetics and the epidemiology of chronic diseases
Washington DC: US Public Health Service. 1965: 61.

7 Meyerowitz S, Jacox R F, Hess D W. Monozygotic twins discordant for rheumatoid arthritis: a genetic, clinical and psychological study of 8 sets. Arthritis Rheum 1968; 1: 1-21.

8 Tarp U, Graudal H K. Seropositive rheumatoid arthritis in monozygotic twin sisters carrying HLA-DR4 or DR4 associated with B27. $\mathcal{F}$ Rheumatol 1989; 16: 1530-2.

9 Lawrence J S H. Rheumatoid arthritis-nature or nurture? [Heberden Oration, 1969]. Ann Rheum Dis 1970; 29: 357-79.

10 Aho K, Koskenvuo M, Tuominen J, et al. Occurrence of rheumatoid arthritis in a nationwide series of twins. f Rheumatol 1986; 13: 899-902.

11 Kasriel J, Eaves L J. The zygotity of twins: further evidence on the agreement between diagnosis by blood group and written questionnaires. F Biosoc Sci 1976; 8: 263-6.

12 Steinbrocker O, Traeger C $\mathbf{H}$, Batterman R C. Therapeutic criteria in rheumatoid arthritis. $\mathcal{F} A M A 1949 ; 140: 659-62$.

13 Arnett F C, Edworthy S M, Bloch D A, et al. The American Rheumatism Association 1987 revised criteria for the classification of rheumatoid arthritis. Arthritis Rheum 1988; 31: 315-24.

14 Norusis M J. SPSS/PC $+V 2 \cdot 0$ base manual. Chicago: SPSS Inc, 1988

15 Ropes M W, Bennett G A, Cobb S, et al. Revision of diagnostic criteria for rheumatoid arthritis. Arthritis Rheum 1959; 2: 16-20.

16 Owen S G, Friesen W T, Roberts M S, et al. Functional capacity and treatment data from a community based study of patients with rheumatoid arthritis. Ann Rheum Dis 1986 45: 293-303.

17 Luukkainen R, Isomaki H, Kajander A. Prognostic value of the type of onset of rheumatoid arthritis. Ann Rheum Dis 1983; 42: $274-5$.

18 Spector T D. Rheumatoid arthritis. In: Hochberg M C, ed. Rheumatic disease clinics of North America: epidemiology of rheumatic disease. Vol. 16 (3). Philadelphia, PA: Saunders, 1990: 513-37.

19 Short C L. The antiquity of rheumatoid arthritis. Arthritis Rheum 1974; 17: 193-6. 